\title{
THE CAUSE OF THE NOVA OUTBURST
}

\author{
S. S T A R R F I E L D \\ Department of Physics, Arizona State University, Tempe, Ariz., U.S.A. and Los Alamos \\ Scientific Laboratory, N.M., U.S.A. \\ W. M. S P A R K S \\ NASA/Goddard Space Flight Center, Greenbelt, Md., U.S.A., \\ and \\ J. W. TRU R A N \\ University of Illinois Observatory, Urbana, Ill. U.S.A.
}

\begin{abstract}
We have evolved a large number of models of thermonuclear runaways in the hydrogen-rich envelopes of $1.00 M_{\odot}$ carbon-oxygen white dwarfs. Models characterized by enhanced CNO abundances satisfy the observations of the common nova outburst. They eject from $10^{27}$ to $10^{29} \mathrm{~g}$ moving with velocities of $200-2400 \mathrm{~km} \mathrm{~s}^{-1}$ : kinetic energies of $10^{44}$ to $10^{45} \mathrm{erg}$. The theoretical light curves are similar to the observed light curves of common novae during the early stages of the outburst. As a consequence of our studies, we can present an explanation for the continuous ejection of mass which is observed for long times after the initial outburst and for the relationship between maximum magnitude and the decline to minimum. We are also now able to present an explanation for the constant luminosity phase of the outburst and the oval shapes of the ejected nebulae.

We have extended our earlier work to models with extreme enhancements of ${ }^{12} \mathrm{C}$ and to models which include the infalling material in the evolution. The extreme ${ }^{12} \mathrm{C}$ studies result in outbursts which reach near supernova proportions while the accretion models result in light curves which resemble observed light curves quite closely. Finally, the accretion studies also demonstrate the need for enhanced abundances.
\end{abstract}

\section{Theory of the Nova Explosion}

\subsection{INTRODUCTION}

Our theory of the nova outburst is based on the accepted model for cataclysmic binary systems: a hot white dwarf accretes hydrogen-rich material supplied by a larger, cooler secondary. The cooler star is losing mass because it fills its Lagrangian lobe and is in a stage of evolution in which it is expanding. This results in a flow of material through the inner Lagrangian point into the lobe surrounding the white dwarf. The cross-section of the white dwarf is small and the material does not directly impact onto its surface but forms a ring of material surrounding it and rotating with high speed.

The nova binary systems were discussed in detail by Kraft (1964) who obtained a period-luminosity relationship for the cataclysmic variables. His relationship implied (with a few exceptions) that the old-novae did not fill their Roche lobes. We have recently re-examined his calculations and find that he used an incorrect formulae for the radius of the Roche lobe - he assumed that the equivalent Roche lobe radius was equal to the barycentric radius of the binary. If we use the correct approximation as given by Paczynski (1971), which assumes synchronous rotation, then the resulting period-luminosity relation shows that the red stars do fill their lobes.

Once it is assumed that the red star fills its lobe, then we must determine the rate of mass loss, since it is this rate which determines the recurrance time scale of the outburst. 
Unfortunately, we cannot be sure about the evolutionary phase of the cool star, although it is generally assumed to be a late-type star currently evolving off the main sequence and expanding. However, it seems unlikely that we are observing cataclysmic binaries in the first phase of mass transfer since the white dwarf must have transferred mass to the cool star in an earlier stage in the evolution of the system. The earlier mass transfer must have affected the evolution of the cool star and possibly the abundances of the elements in the material now being transferred back to the white dwarf.

Another consideration is the rate of mass transfer. The rate calculated from studies of the accretion shock in DQHer was $10^{-8} M_{\odot} \mathrm{yr}$ (Starrfield, 1970), while Herbst et al. (1974) determined a rate of $5 \times 10^{-9} M_{\odot} \mathrm{yr}^{-1}$ from studies of variations in the 71 second pulsation period of DQ Her. Walker and Chincarini (1968) found a variable orbital period for SS Cygni and attributed it to a mass loss rate of $10^{-7} M_{\odot} \mathrm{yr}^{-1}$. However, none of these studies is conclusive because the old novae systems are too complicated for the results noted above to have much value. For example, there are other sources of light in DQ Her besides the accretion shock, and it now appears that the $71-\mathrm{s}$ variation may be actually a 142-s rotation and not a pulsation (Swedlund et al., 1974). In addition, the mass transfer rate is a very complicated function of the orbital period change. If these values of the mass transfer rate were accurate, we would expect the ring surrounding the white dwarf to have a mass of about the same order $-10^{-7}$ to $10^{-8} M_{\odot}$. However, Smak (1971) has determined that the ring in $\mathrm{U} \mathrm{Gem}$ is quite massive $\sim 10^{-4} M_{\odot}$. If this result is generally applicable, which is unlikely, then it is hard to understand how DQ Her, transferring mass at a rate of $10^{-8} M_{\odot} \mathrm{yr}^{-1}$, could have re established a disc of even $10^{-6}$ or $10^{-5} M_{\odot}$ in the $40 \mathrm{yr}$ since its outburst. A possible explanation is that the rings of the common novae are not as massive as the rings of the dwarf novae.

We then assume that the material reaches the white dwarf and forms a layer on the surface. As the inflow proceeds the deeper regions are compressed and heated until thermonuclear temperatures are reached and nuclear burning begins. The original hydrostatic study of accretion onto a white dwarf was discussed by Giannone and Weigert (1967) and Rose (1968) who found that a white dwarf could accrete $10^{-3} M_{\odot}$ before the energygeneration time scale had decreased below the mass accretion time scale. Aside from minor variations in the envelope mass, $10^{-3} M_{\odot}$ is the value that we have used in all our published studies. More recently, Taam and Faulkner (1975) have studied mass accretion onto a $1.00 M_{\odot}$ white dwarf and achieved thermonuclear runaways with envelope masses of $10^{-4} M_{\odot}$. We expect no major changes in our results but it now seems useful to redo some of our earlier work with smaller envelope masses.

We, therefore, take as our model for the object on which the outburst takes place a white dwarf with a thin envelope of hydrogen rich material. In our initial studies (Starrfield et al., 1972, Paper I; Starrfield et al., 1974a, Paper II) we assumed the envelope was in place and in equilibrium; and followed the development of the thermonuclear runaway with time using a Lagrangian, fully implicit, hydrodynamic computer code incorporating a nuclear reaction network (Kutter and Sparks, 1972). Our major discovery was that we could reproduce the gross features of the nova outburst as long as the hydrogen envelope was overabundant in the CNO nuclei. Our models with enhanced ${ }^{12} \mathrm{C}$ and ${ }^{16} \mathrm{O}$ ejected $10^{28}$ to $10^{29} \mathrm{~g}$ moving with speeds of 200 to $4000 \mathrm{~km} \mathrm{~s}^{-1}$, kinetic energies of $10^{45} \mathrm{erg}$. In addition, these same models produced total energies of $10^{46}$ to $10^{47} \mathrm{erg}$ in excellent agreement with the UV observations (Gallagher and Code, 1974) 
and the IR observations (Geisel et al., 1970) of FH Ser. Those models with no nuclear enhancement (above solar) did not eject any material and their peak kinetic energy, $10^{42} \mathrm{erg}$, was insufficient to eject the ring of material surrounding the white dwarf.

In the following sections we will present a more detailed discussion of the thermonuclear runaway model of the nova outburst. We begin, in Section 1.2 by re-deriving the period-luminosity relationship for the cataclysmic variables (Kraft, 1964) and show that the secondaries do fill their lobes. In the following section we discuss the hydrostatic studies of accretion onto white dwarf and their connection with our hydrodynamic studies: In succeeding sections we describe our model for the outburst and the need for enhanced abundances in the hydrogen envelope; the evolution of models with different chemical abundances; and the implications of our results for observations of the nova outburst.

\subsection{A PERIOD LUMINOSITY RELATIONSHIP FOR THE CATACLYSMIC VARIABLES}

In an important paper Kraft (1964) studied the spectral characteristics of 10 old novae and found additional evidence to support the hypothesis that all old novae are binaries. In this paper he derived a period-luminosity relation for these variables based on Kepler's Law and the assumed fact that the cool secondary filled its Roche lobe. He used this expression to show that either the red stars were underluminous for their masses or that they did not fill their Roche lobes. Although these conclusions have been completely forgotten and it is generally assumed that the red stars fill their lobes, it is the purpose of this section to point out an error in his derivation and present a revised expression which implies neither of the above conclusions.

The difficulty arose in Equation (4) of Kraft's (1964) paper which was an expression relating the radius of the red star, the binary period, and the mass ratio. He eliminated the semi-major axis by assuming that the red star extended to the surface of the Roche lobe. However, he took as the value for the Roche lobe radius the barycentric radius, which is incorrect. A better value, correct for a large range of mass ratios, can be found in Paczynski (1971). In the derivation to be presented here we shall use

$$
\frac{r_{R}}{A}=\frac{2}{3 \frac{4}{3}}\left(\frac{M_{R}}{M_{R}+M_{\mathrm{B}}}\right)^{\frac{1}{3}}
$$

which is reasonably accurate and simple. Following Kraft (1964), we write

$$
L_{R}=k 4 \pi R_{R}^{2} \sigma T_{\mathrm{e}}^{4}
$$

where $k$ allows for any failure of this expression. For example, Kraft found that $k=0.1$. Substituting (1) and (2) into Kepler's Law we find that

$$
P^{2}=\frac{3^{4} \pi^{2}}{2}\left(\frac{L}{L_{\odot}}\right)^{\frac{3}{2}} k^{-\frac{3}{2}} R_{\odot}^{3}\left(\frac{T_{\mathrm{e}}}{T_{\mathrm{e}}}\right)^{-6} \frac{1}{G M_{R}}
$$

where $P$ is the orbital period. Taking the common logarithm and converting the expression in to magnitudes we arrive at:

$$
M_{\nu}(\mathrm{red})=-\frac{10}{3} \log P-\frac{5}{2} \log k-\frac{5}{3} \log M(\mathrm{red})
$$




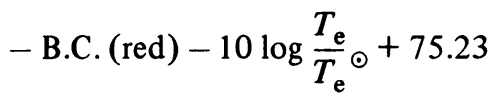

In this expression the constant is expressed in cgs units while 'B.C.' stands for the bolometric correction. Notice that this formula differs from Kraft's in the term's involving the mass of the red star and in the magnitude of the numerical constant. Continuing, we take values of the parameters from Allen (1963) for stars with masses of $0.2 M_{\odot}, 0.5$ $M_{\odot}, 0.7 M_{\odot}$, and $1.0 M_{\odot}$ and average the values. The result is that

$$
M_{v}(\mathrm{red})=-2.5 \log k-\frac{10}{3} \log P+11.1
$$

with $P$ expressed in hours. The value of the numerical constant is 2.5 mag larger than Kraft's so that his line for $k=0.1$ is actually the line for $k=1$. This removes the discrepancy and it can be assumed that the red stars fill their lobes.

\subsection{HYDROSTATIC STUDIES OF ACCRETION ONTO WHITE DWARFS}

Our modern theoretical studies of the nova outburst date from the work of Giannone and Weigert (1967) who evolved a $0.5 M_{\odot}$ white dwarf accreting hydrogen rich material and showed that a thermonuclear runaway could be initiated in the envelope. Their work was followed by Rose (1968), Redkoborodyi (1972), and Taam and Faulkner (1975). These authors studied material accreting onto $0.5 M_{\odot}, 0.75 M_{\odot}$, and $1.0 M_{\odot}$ white dwarfs and also found that thermonuclear runaways occurred in the envelope. They used rates of infall varying from $10^{-5} M_{\odot} \mathrm{yr}^{-1}$ (Rose, 1968) to $10^{-11} M_{\odot} \mathrm{yr}^{-1}$ (Giannone and Weigert, 1967); all cases except the last one resulted in a thermonuclear runaway. A summary of their results is presented in Table $\mathrm{I}$. It is clear from examining the table that these hydrostatic

TABLE I

\begin{tabular}{|c|c|c|c|c|c|c|c|c|}
\hline $\begin{array}{l}M \\
\left(m_{\odot}\right)\end{array}$ & $\begin{array}{l}\log L / L_{\odot} \\
\text { (initial m }\end{array}$ & $\begin{array}{l}\log T_{\mathrm{e}} \\
\text { odel) }\end{array}$ & $\begin{array}{l}\dot{M} \\
\left(m_{\odot} \mathrm{yr}^{-1}\right)\end{array}$ & $\begin{array}{l}M_{\mathrm{e}} \\
\left(m_{\odot}\right)\end{array}$ & $\begin{array}{l}\log T_{\mathrm{s}} \\
(\operatorname{deg} \mathrm{K})\end{array}$ & $\begin{array}{l}\log \rho_{\mathrm{s}} \\
\left(\mathrm{gm} \mathrm{cm}^{-3}\right)\end{array}$ & $\begin{array}{l}\tau_{\text {run }} \\
(\mathrm{yr})\end{array}$ & Reference \\
\hline $\begin{array}{l}0.5 \\
0.5 \\
0.5 \\
0.75 \\
0.75\end{array}$ & $\begin{array}{c}-1.507 \\
-3.480 \\
-1.57 \\
1.80 \\
1.80\end{array}$ & $\begin{array}{l}4.281 \\
3.804 \\
4.281 \\
5.02 \\
5.02\end{array}$ & $\begin{array}{r}10^{-9} \\
10^{-9} \\
10^{-9} \\
7 \times 10^{-8} \\
1 \times 10^{-5}\end{array}$ & $\begin{array}{l}6.4 \times 10^{-4} \\
1.3 \times 10^{-3} \\
8 \times 10^{-4} \\
1.8 \times 10^{-4} \\
3.5 \times 10^{-4}\end{array}$ & $\begin{array}{l}7.73 \\
7.60 \\
7.47 \\
7.85 \\
7.85\end{array}$ & $\begin{array}{l}2.793 \\
3.349 \\
2.99 \\
1.60 \\
2.14\end{array}$ & $\begin{array}{l}6.42 \times 10^{5} \\
1.3 \times 10^{6} \\
8.1 \times 10^{5} \\
4 \times 10^{2} \\
1.4 \times 10^{1}\end{array}$ & $\begin{array}{l}\text { GW } \\
\text { GW } \\
\text { RE } \\
\text { RO } \\
\text { RO }\end{array}$ \\
\hline $\begin{array}{l}1.0 \\
1.0\end{array}$ & $\begin{array}{l}-2.29 \\
-3.27\end{array}$ & $\begin{array}{l}4.23 \\
3.99\end{array}$ & $\begin{array}{l}10^{-10} \\
10^{-10}\end{array}$ & $\begin{array}{l}1 \times 10^{-4} \\
2 \times 10^{-4}\end{array}$ & $\begin{array}{l}7.39 \\
7.51\end{array}$ & $\begin{array}{l}3.79 \\
3.80\end{array}$ & $\begin{array}{l}1.5 \times 10^{6} \\
3.4 \times 10^{6}\end{array}$ & $\begin{array}{l}\text { TF } \\
\text { TF }\end{array}$ \\
\hline
\end{tabular}

Hydrostatic accretion onto white dwarfs

Notes:

GW = Giannone and Weigert (1967)

$\mathrm{RE}=$ Redkoborodyi (1972)

RO = Rose

$\mathrm{TF}=$ Taam and Faulkner (1975)

$M_{\mathrm{e}}=$ envelope mass

$T_{\mathrm{s}}=$ the temperature in the shell source for the last quoted model.

$\rho_{\mathrm{S}}=$ the density in the shell source for the last quoted model. 
studies provide excellent initial models for the hydrodynamic studies (in which mass accretion over long time scales cannot presently be included). We can use these studies to determine the amounts of mass that can be accreted in the envelope before a thermonuclear runaway results and, in addition, the time scales to the start of the thermonuclear runaway. At the present time there is no way for us to obtain an accurate value of the time between outbursts from our hydrodynamic studies since we begin with the envelope in place and in equilibrium on the white dwarf.

The results of the hydrostatic studies also reveal that the amount of mass accreted before the flash is a function of both the white dwarf mass and its initial luminosity. The more massive a white dwarf the less massive the envelope needed to produce a thermonuclear runaway. This is attributable to the larger gravity and pressure gradient in the more massive white dwarfs which causes the bottom of the accreted material to reach thermonuclear densities with a relatively smaller amount of material in the envelope. The dependence on the initial luminosity results from the fact that, for a white dwarf of a given mass, the allowed mass of the accreted envelope increases as the luminosity decreases. This is because the temperature at the bottom of the envelope is a function of the luminosity of the white dwarf for low mass accretion rates, and because the time to peak burning is a strong function of the temperature (Paper II). The lower the initial luminosity and therefore the internal temperature, the longer the runaway time and the larger the mass that can be accreted in that time.

There is a complicating factor: the rate of mass accretion. In the case with the largest rate of mass accretion, $10^{-5} M_{\odot} \mathrm{yr}^{-1}$ (Rose, 1968), more mass was accreted than we would expect for the given luminosity and white dwarf mass. Unfortunately, in this case the neglect of the infall energy probably causes the models to be totally unrealistic. We would expect the envelope to expand and become non-degenerate preventing a violent flash (Starrfield et al., 1974d).

We feel that the hydrostatic studies provide us with valid useful initial models. We can use their results to predict the starting conditions for our hydrodynamic studies realizing, of course, that beginning with a model that has the envelope in place and in equilibrium will not provide us with the correct time scales for the evolution to the outburst. In addition, since the hydrostatic studies start with no hydrogen in the envelope they will have long evolution time even at fairly high initial luminosities contrary to the results of our hydrodynamic studies (Paper II).

Up to the present time we have used envelope masses of $10^{-3} M_{\odot}$ justifying this amount by the work of Giannone and Weigert (1967). In the future, since we are studying white dwarfs with larger masses, we shall reduce the envelope mass to $10^{-4} M_{\odot}$ or less.

\subsection{ROSE AND SMITH'S MODEL FOR THE OUTBURST}

Rose and Smith (1972) proposed a model for the nova outburst based on hydrodynamic studies both of a shock moving through an envelope and of stellar pulsation. Unfortunately they used an incorrect formulation for the rate of energy generation which invalidated their primary results. Furthermore, their pulsation study used a spherical piston at the bottom of the envelope which renders their pulsation results uninterpretable.

Their expression for the rate of energy generation was obtained from an expression in Clayton (1968) for the equilibrium rate of the $\mathrm{CN}$-cycle. This formula is not valid until the evolution time in the model reaches $10^{5} \mathrm{yr}$, although they used it for their stated 
time scales of $10^{4} \mathrm{~s}$. In addition, they took the rate from this expression and multiplied it by an arbitrary factor which increased with time. This procedure does produce a shock which ejects some material but it is not possible to justify this procedure with a physical mechanism. Moreover, their envelope temperatures exceeded $10^{8} \mathrm{~K}$ and their approximation does not correctly model the behavior of the $\beta^{+}$-unstable nuclei which become abundant at this temperature.

Their second attempt to produce an outburst was based on the pulsation study of Rose (1968). However, because they were unable to produce pulsations as a result of their evolution, they put a spherical piston into the envelope in order to produce pulsations. Nevertheless, no mass loss resulted from this procedure even when the piston was being driven to produce shocks. The difficulty with using a piston is that a large number of modes are excited at the same time. The early cepheid studies using this approach were not even published because it was found that the results depended only upon the details of the piston's period and amplitude (A. N. Cox, 1975; private communication).

\subsection{THE ENHANCED CNO MODEL FOR THE OUTBURST}

Since the idea that the CNO nuclei must be enhanced to produce an outburst has met with a great deal of astronomical resistance, in Paper II we presented an order of magnitude argument which supported our results. Here we present another argument for enhanced abundances. As the nova outburst takes place on a white dwarf with a radius of about $10^{9} \mathrm{~cm}$, the energy $G M^{2} / R$ required to eject material to infinity is $10^{17} \mathrm{erg} \mathrm{gm}^{-1}$. This does not include the energy needed to eject the accretion disc which is moving supersonically around the white dwarf. Normally in a low temperature region the CNO Cycle releases $6 \times 10^{18} \mathrm{erg} \mathrm{gm}^{-1}$, a value which assumes that all the reactions are proceeding in equilibrium. However, this cannot be the case in a thermonuclear runaway in which most of the temperature rise occurs in the last few minutes of the outburst. At this time the only reactions that can proceed at a sufficient rate are the proton-capture reactions: $\left.{ }^{12} \mathrm{C}(\mathrm{p}, \gamma)\right)^{13} \mathrm{~N},{ }^{13} \mathrm{C}(\mathrm{p}, \gamma){ }^{14} \mathrm{~N},{ }^{14} \mathrm{~N}(\mathrm{p}, \gamma){ }^{15} \mathrm{O},{ }^{15} \mathrm{~N}(\mathrm{p}, \alpha){ }^{12} \mathrm{C}$, and $\left.{ }^{13} \mathrm{~N}(\mathrm{p}, \gamma)\right)^{14} \mathrm{O}$ which release only about $10^{15} \mathrm{erg} \mathrm{gm}^{-1}$ (for a solar mixture). If the envelope has a mass of $10^{-3} \mathrm{M}_{\odot}$, then the maximum energy these reactions can release is $10^{45} \mathrm{erg}$. However, we need $10^{45}$ to $10^{46} \mathrm{erg}$ to eject the expanding nebulae which are observed to have masses of $10^{-4}$ to $10^{-5} M_{\odot}$, expansion speeds of $10^{3} \mathrm{~km} \mathrm{~s}^{-1}$, and kinetic energies of $10^{44}$ to $10^{45} \mathrm{erg}$. In addition Hazlehurst (1962) and Sparks (1969) have shown that the conversion from internal energy to kinetic energy is only about 1 to $10 \%$ efficient requiring that $10^{46}$ to $10^{47}$ erg be involved in the outburst. Therefore, our energy argument requires enhancements of at least a factor of 10 to produce the energy observed in the nova outburst.

We have as yet no explanation for the source of the CNO nuclei. The most favorable response has been to our suggestion (Paper II) that the outburst is occurring on a carbon white dwarf and some protons are being mixed into the outer edges of the core. This idea was expanded on by Hoyle and Clayton (1974) who suggested that the material was accreted in the form of 'blobs' which penetrate deeply into the white dwarf. Their theory was in opposition to the usual assumption of a steady 'rain' of material on to the surface. However, a recent two-dimensional calculation has shown that the blobs will not penetrate into the surface; the density gradient is too large (Cloutman and Starrfield, 1975).

A more plausible idea is that the outer edge of the white dwarf becomes convectively 
unstable during the evolution and mixes the accreted protons into the carbon core. Support for this suggestion has recently appeared in a paper by Lamb and Van Horn (1975). They have evolved a $1.00 M_{\odot}$, pure carbon, white dwarf past the onset of crystallization and find that the outer layers become convectively unstable at a luminosity of $10^{-2}$ $L_{\odot}$ and the depth of the convective region increases as the dwarf's luminosity decreases. If such a white dwarf were a member of a close binary system and mass transfer onto the surface did not begin until it had developed a deep convective envelope, then it seems possible that convection is our enhancement mechanism. Very recently, Van Horn and Colvin (1975, private communication) have accreted hydrogen onto a $1.00 M_{\odot}$ dwarf with a convective envelope and find that hydrogen is carried to the bottom of the unstable region and gradually 'poisons' the convection from the bottom up. It would be very useful to use their composition profiles in our studies. However, even if this idea is correct it is still left to be determined how a $1.00 M_{\odot}$ carbon white dwarf can be produced in a close binary system.

If we assume that the CNO nuclei have been enhanced in the envelope, then the increased abundances will have the following effects. During the initial temperature rise most of the energy generation comes from proton captures onto ${ }^{12} \mathrm{C},{ }^{13} \mathrm{C},{ }^{14} \mathrm{~N}$, and ${ }^{15} \mathrm{~N}$. Then, as the time scale for the energy generation decreases, the $\beta^{+}$-unstable nuclei become the most abundant of the CNO nuclei in the envelope. This halts a further rise ir energy generation since no further proton captures can occur until the $\beta^{+}$-unstable nuclei decay. Therefore, in an outburst, the peak rate of energy generation depends only on the total abundance of the CNO nuclei in the envelope.

The further evolution of the envelope is determined by the $\beta^{+}$-decay time scale of ${ }^{13} \mathrm{~N},{ }^{14} \mathrm{O}$, and ${ }^{15} \mathrm{O}$, because it is the decay of these nuclei which provides the energy necessary to eject the material observed in the nova outburst and provides the energy needed to produce the sudden rise in luminosity which is characteristic of novae light curves. However, the second rise is probably caused by equilibrium $\mathrm{CN}$ burning in the remaining envelope at later stages in the outburst (Sparks et al., 1976, in press). In addition, the $\beta^{+}$-unstable nuclei will decay when the envelope has already expanded to $10^{10} \mathrm{~cm}$ when less energy is needed to eject the shell. Finally, since the temperature in the envelope will now be too low for further proton captures to occur; the final abundances will not be representative of equilibrium CNO burning at any temperature. However, because the ejected material must also carry the accretion disc along with it, the observed abundances will reflect a mixture of the envelope and disc and will not agree in detail with our predictions.

In fact, the observations show that the CNO abundances in the novae ejecta are non-solar and definitely enhanced. The earliest report was from a study of Pottasch (1958) who found that carbon, nitrogen, and oxygen were overabundant in the nebular spectrum. More recently Antipova (1974) Ruusalepp and Ludd (1970), Sneden and Lambert (1975), Anderson and Gallagher (1975, private communication), and Sanyal and Robbins (1975, private communication to SGS) have reported enhanced abundances and even non-solar isotopic ratios in novae ejecta. The present observational result appears to be that the CNO nuclei are enhanced in the novae. This means that they must have been enhanced before the outburst; since the CNO reactions only redistribute the nuclei, they do not produce new CNO nuclei. Therefore, our major observational prediction is in agreement
with the observations. 


\subsection{EVOLUTION OF THE MODELS}

In our initial studies we varied the luminosity of the white dwarf, the degree of ${ }^{12} \mathrm{C}$ and ${ }^{16} \mathrm{O}$ enhancement, and to a lesser degree, the hydrogen envelope mass (Paper I, Paper II). We have since evolved models with different white dwarf mass (Starrfield et al., 1974b, Paper III; Starrfield et al., 1974c, Paper IV; Starrfield et al., 1975, Paper V) extreme variations of the ${ }^{12} \mathrm{C}$ abundance (Paper IV and V), and included the effects of the infalling material on the evolution of the outburst (Starrfield et al., 1974d, Paper VI). In these studies we found that the principal effect of variations in luminosity was on the time to peak temperature - the lower the luminosity the longer the evolution time. We have proposed that this result is responsible for Kukarkin and Parenago's relationship. We also found that the degree of enhancement of the CNO nuclei was directly related to the strength of the outburst in that the strength (or violence) increased as we increased the amount of CNO nuclei in the hydrogen envelope. We, therefore, proposed that the abundance enhancement was one of the factors affecting the differences between the fast and slow novae.

In fact, as further evidence for our mechanism, we studied various models with only a solar abundance of CNO nuclei in the envelope. In no case did the energy produced during the outburst exceed $10^{41} \mathrm{erg}$ and no material was ejected. The decay of the $\beta^{+}$-unstable nuclei was sufficient to expand the surface layers only to about $10^{10} \mathrm{~cm}$ but the major increase in luminosity was caused by equilibrium CNO burning in the shell source. Figure 1 shows the light curve for one of these models; it looks completely unlike that of the nova.

The models in Paper II that most closely satisfied the observed conditions for the nova outburst were those with the deepest hydrogen rich zone enhanced to $20 \%$ carbon and

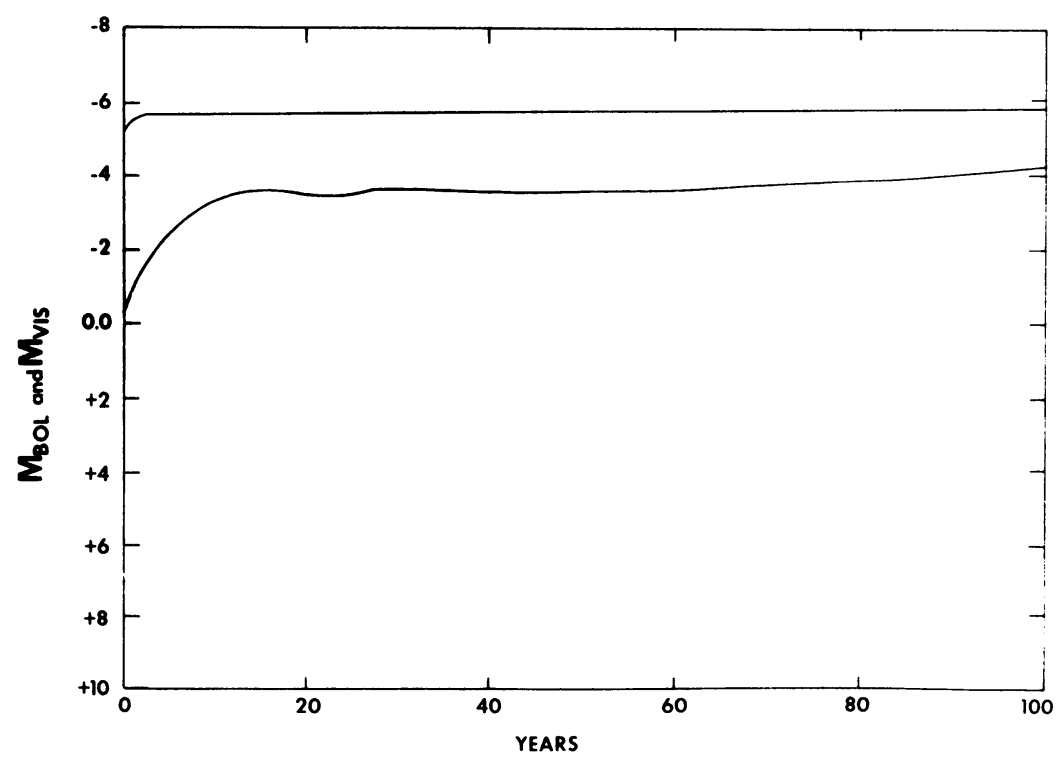

Fig. 1. The light curve for a model with $12 \%$ carbon and oxygen in the deepest hydrogen rich zone. The upper curve is $M_{\mathrm{Bol}}$ and the lower curve is $M_{\mathrm{Vis}}$. After a few months the white dwarf is evolving on a nuclear burning time scale. 
$20 \%$ oxygen by mass. If we choose a low enough luminosity, $L<10^{-3} L_{\odot}$, then the evolution time of these models is long enough so that a thick degenerate envelope can be accreted. One such model, model 7 of Paper II, took about $10^{5} \mathrm{yr}$ for the temperature in the shell source to reach 30 million deg. It then flashed to a peak burning rate of $4 \times 10^{16} \mathrm{erg} \mathrm{gm}^{-1} \mathrm{~s}^{-1}$ and a peak temperature of 221 million deg. Figure 2 shows how the temperature in the shell source varied with time while Figure 3 shows the variations of the nuclear abundances. The 'sawtooth' pattern is caused by oscillations of the envelope. These oscillations appear right after the time of peak burning in the shell source but are quickly damped by the formation of shocks as the material falls back onto the dwarf. The variations in the radii of 3 shells at the time of peak temperature is shown in Figure 4.

This model ejects $3 \times 10^{29} \mathrm{~g}$ as the $\beta^{+}$-unstable nuclei decay and release more than $10^{47} \mathrm{erg}$ into the envelope. The expanding shells quickly reach velocities of a few hundred to a few thousand $\mathrm{km} \mathrm{s}^{-1}$; kinetic energies of $10^{45} \mathrm{erg}$. The light curve of one such model is shown in Figure 5. This figure also shows the effect of chemical composition on the light curve. The Aller mix has a higher $Z$ and so the opacity is higher keeping the nebula optically thick for a longer time. The expanding shells are also significantly enhanced in the CNO nuclei with the isotopic ratios showing strong evidence of nuclear burning far from equilibrium conditions.

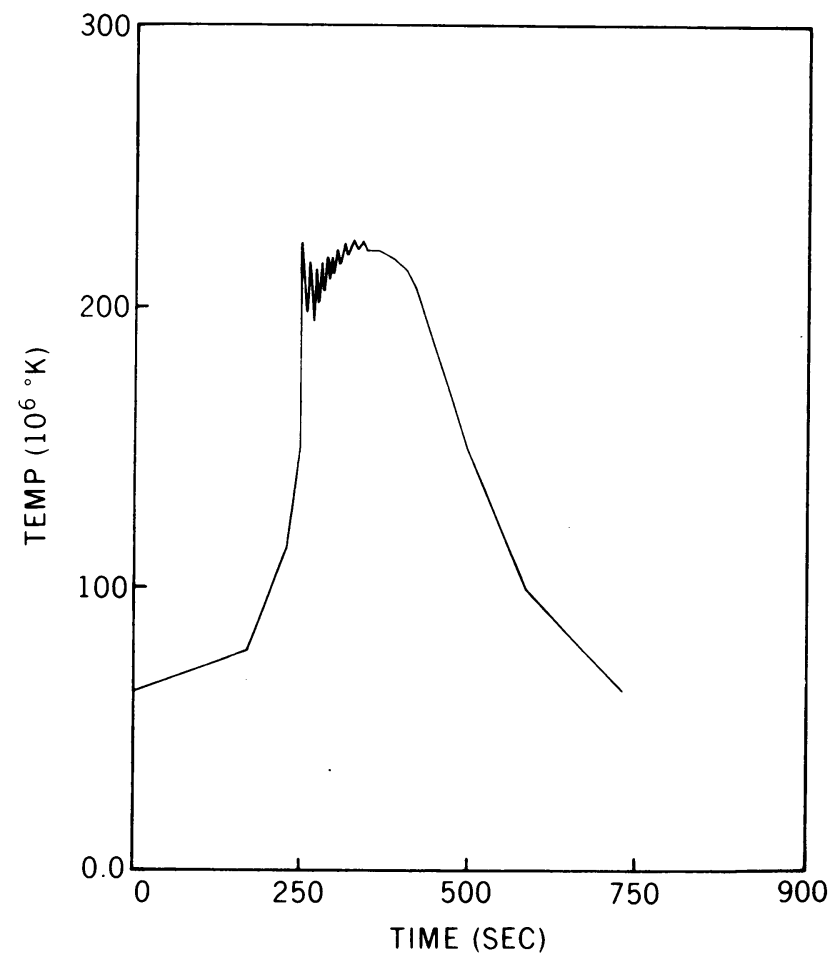

Fig. 2. The temperature in the shell source as a function of time for a model with $20 \%$ carbon and oxygen in the deepest hydrogen rich zone. This model is called number 7 in Paper II. The oscillations are caused by mass motions in the envelope and are damped by the formation of shocks. 
In addition to the other successes of our evolutionary studies, it now appears that we can point to the cause of the continuous ejection phase of the outburst and relate it to the differences between the fast and slow novae. As we have already reported (Paper II, Paper III), only about $10 \%$ of the hydrogen envelope is ejected during the hydrodynamic phase of the outburst. The outward velocity of the material in the deeper zones quickly drops and this material returns to hydrostatic equilibrium. However, the shell source has not been extinguished and, in fact, is now producing energy at a rate of $10^{8}$ to $10^{9} \mathrm{erg}$ $\mathrm{gm}^{-1} \mathrm{~s}^{-1}$. Over the next few days the entire envelope becomes convective and the effective temperature of this remnant climbs to $10^{5} \mathrm{~K}$, although it will remain hidden until the expanding nebula has become optically thin. We have proposed that it is this hot remnant that is responsible for the UV bright source reported by Gallagher and Code (1974).

The radius of the hydrostatic remnant extends to $10^{10}$ to $10^{12} \mathrm{~cm}$ depending upon the amount of mass remaining in the hydrogen envelope of the white dwarf. The greater the envelope mass, the larger the radius of the remnant. The size of this remnant implies that the material will extend past the Roche lobes of the binary system for all but the binaries with the longest periods. Therefore, the final stage of the nova outburst will involve a 'blue horizontal-branch' object with a close binary revolving around inside of it. This does

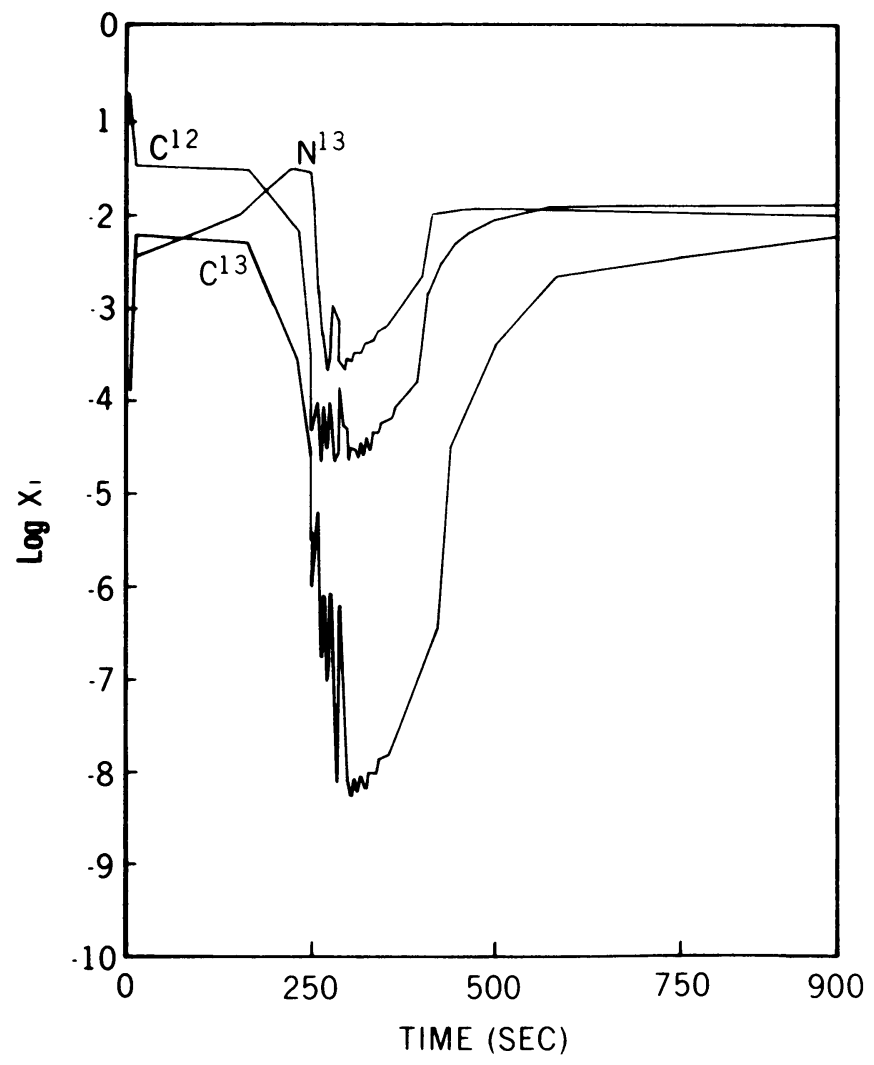

Fig. 3a. 


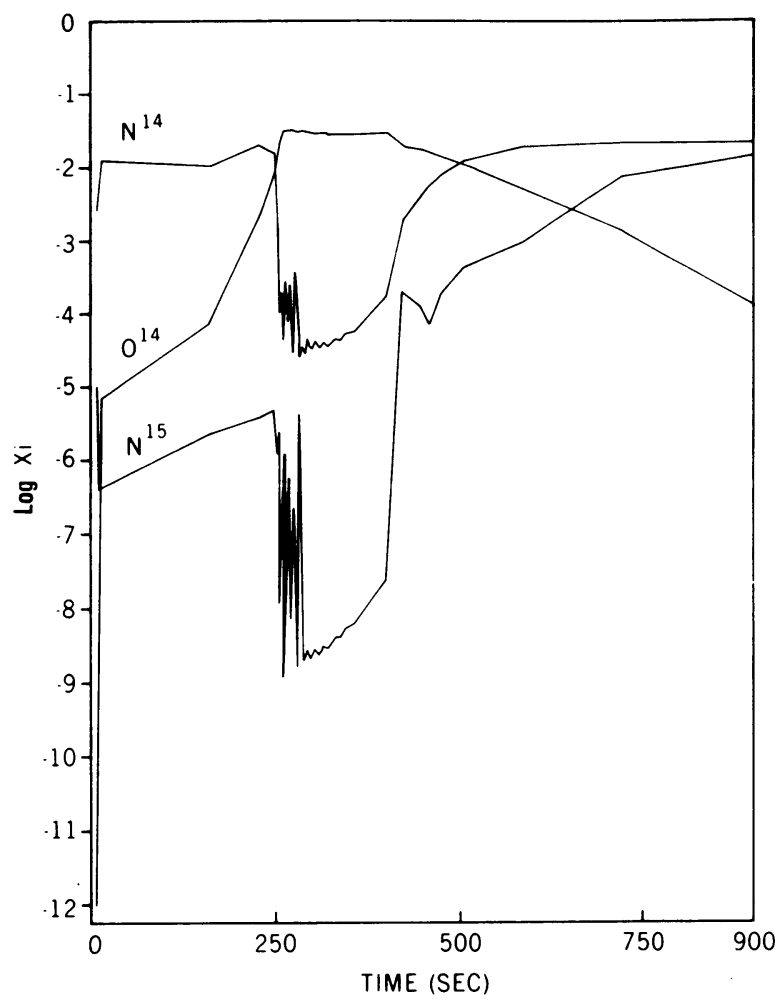

Figs. $3 \mathrm{a}$ and $3 \mathrm{~b}$. The abundance by weight of the listed nuclei as a function of time for the deepest hydrogen-rich shell in the same model as depicted in Figure 2. Changes in the temperature gradient cause convection to turn on and off mixing in fresh nuclei from time to time. Notice the difference in behavior between the nuclei able to proton capture and ${ }^{14} \mathrm{O}$ which can only $\beta^{+}$-decay. The initial point for each nucleus marks its abundance at the beginning of the evolution, not at time zero on the graph.

not appear to be a stable situation (Paczynski, 1976) and mass loss should occur at a large rate until the equilibrium radius of the remnant has again decreased to within the Roche lobe of the binary. It is possible that the radiation driven mass loss mechanism of Lucy and Solomon (1970) can be applied to these hot remnants, or the instability described by Bath (1974) is also relevant since Bath's results apply directly to stars with convective envelopes which extend to or past the Roche lobe.

One of the more important recent developments is the proposal that the diffuseenhanced and orion spectra of the novae are associated with the remnant, if not caused directly by mass loss from the remnant (Gallagher and Starrfield, 1976). We obtained this result as a consequence of the earlier finding of Gallagher and Code (1974) that there is a constant luminosity, high temperature source inside the expanding nebulae of FH Ser. If we assume the luminosity of the remnant is constant and the temperature is increasing, although the luminosity remains constant, then the visual magnitude will decline purely from the increasing bolometric correction. In order to demonstrate this we now assume that the diffuse-enhanced spectrum is produced by a 'stellar' atmosphere and the observed 


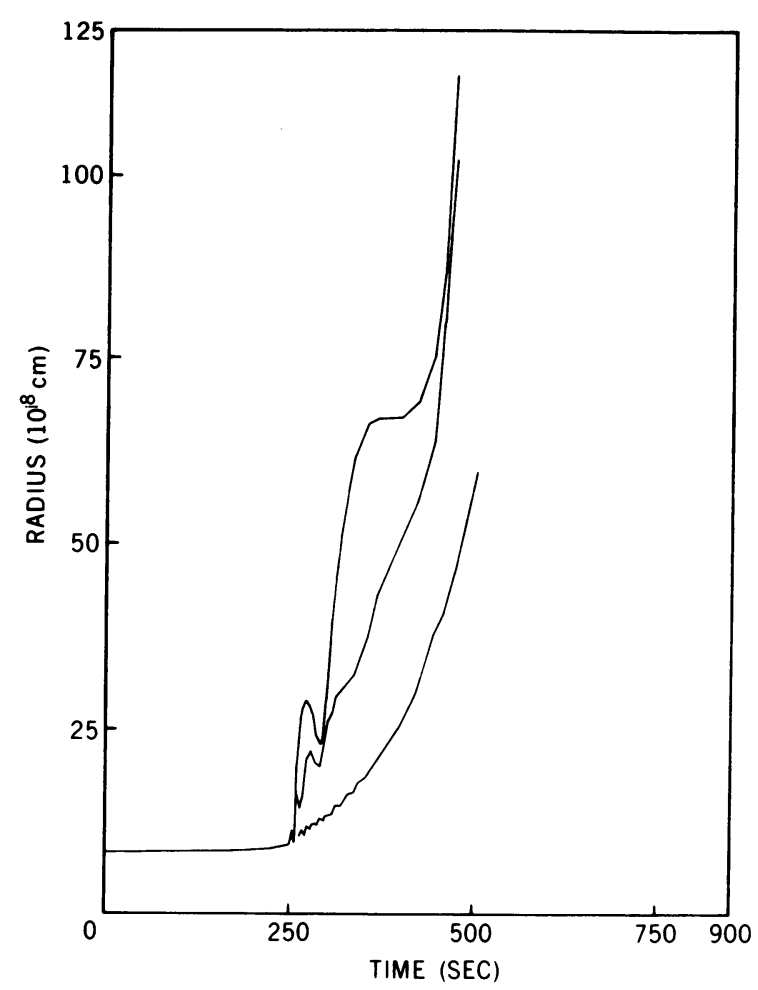

Fig. 4. The radii of three shells as a function of time for the same model and time scale as in Figures 2 and 3 . The top curve shows the surface of the dwarf.

lines can be used to obtain an effective temperature. The observed maximum luminosity and the effective temperature derived for FH Ser imply an object with a radius of about $10^{11}$ to $10^{12} \mathrm{~cm}$ - the predicted radius of the theoretical remnants. This procedure seems reasonable since the bolometric correction for stars of the given temperature is equal to the difference in magnitude of FH Ser at the time of maximum light and at the time when the diffuse-enhanced spectrum is prominent. If we apply this same argument to the orion spectrum, which contains lines of higher excitation, we obtain a higher effective temperature and, therefore, a larger bolometric correction. As before, the visual magnitude, when the orion spectrum is prominent, plus the bolometric correction is equal to the magnitude of the nova at maximum. The derived radius; from the luminosity at maximum and the derived effective temperature, imply that this object has a radius of $10^{10}$ to $10^{11} \mathrm{~cm}$, the radius of the Roche lobes of the cataclysmic binaries. Since the orion spectrum appears at a later stage in the evolution of the nova, we suggest that the change from one spectrum to another is connected with the equilibrium radius of the remnant shrinking within the Roche lobe as the remnant loses mass. Neglected in this discussion is that both these spectra are made up of systems of absorption lines all blue-shifted by large velocities with respect to the principal emission lines. In addition, while the blue shifts amount to thousands of kilometers per second, the halfwidth of the lines are only 


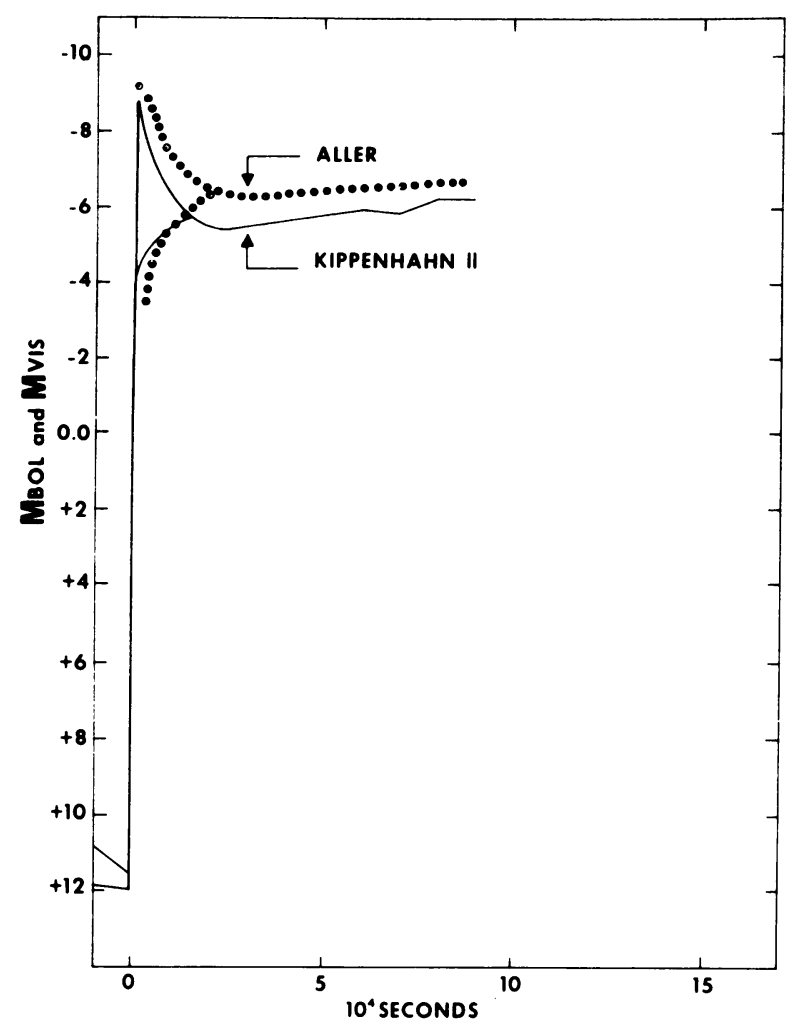

Fig. 5. A comparison of the light curves for the same model shown in Figures 2 to 4 and an identical model computed with a different composition. 'Aller' refers to an opacity table given in Cox and Stewart (1965) while Kippenhahn II refers to another opacity table in the same publication. In each

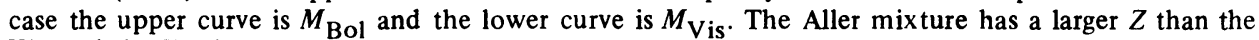
Kippenhahn II mixture.

$30-50 \mathrm{~km} \mathrm{~s}^{-1}$. This behavior is astrophysically similar to the absorption systems observed in QSO's!

In other recent work we have found that the mass of the white dwarf does affect the characteristics of the nova phenomena. We have studied models with $0.5 M_{\odot}$ and a variety of CNO enhancements and models with $1.1 M_{\odot}$ and $1.25 M_{\odot}$ with extreme carbon enhancement (Paper III, IV, V). We had the most difficulty in producing an outburst on the $0.5 M_{\odot}$ white dwarf. In fact, we had to quadruple the envelope mass (to $10^{31} \mathrm{~g}$ ) and enhance the CNO nuclei, to a degree that exceeded the $1.00 M_{\odot}$ models, before ejection occurred and an outburst resulted. This is because the larger radius and smaller mass reduces the pressure gradient and the degree of degeneracy at a given depth in the star as compared to the $1.00 M_{\odot}$ models. With a smaller degeneracy, the peak burning rates and peak temperatures are smaller in the lower mass white dwarfs. We have suggested that one of the factors producing the variation among the speed classes is the mass of the white dwarf on which the outburst takes place.

It is not possible to directly compare the results for the more massive white dwarfs with the studies at $1.00 M_{\odot}$ since we have evolved the $1.1 M_{\odot}$ and $1.25 M_{\odot}$ models with 
extreme overabundances of ${ }^{12} \mathrm{C}$ in the envelope. The massive white dwarfs were studied after Hoyle and Clayton (1974) suggested that nova models with equal amounts of ${ }^{12} \mathrm{C}$ and ${ }^{1} \mathrm{H}$ in the envelope could produce $s$-process nucleosynthesis. We evolved two models with variations of envelope composition and found that, in fact, the $1.25 M_{\odot}$ sequence produced neutrons in the deepest shell of the envelope. However, the rate was too large to be called an $s$-process. More interesting, these models were the most violent that we have evolved. They produced peak temperatures of over $10^{9} \mathrm{~K}$ in the envelope and burning rates which exceeded $10^{21} \mathrm{erg} \mathrm{gm}^{-1} \mathrm{~s}^{-1}$. A shock resulted and ejected more than $10^{29} \mathrm{~g}$ moving with speeds of up to $90000 \mathrm{~km} \mathrm{~s}^{-1}$. The kinetic energies in the expanding shell was nearly $10^{47}$ erg while the peak absolute visual magnitude was $\sim-12$ (Figure 6 ). Although we hesitate to compare these values with observed novae, CP Pup had a range of nearly 17 mag and reached an absolute visual magnitude of -10.5 (Payne-Gaposchkin, 1957). Unfortunately, its observed velocities were very much smaller than our studies would have predicted. Possibly the material expanding off the white dwarf ran into the ring.

We have also been able to include the infalling material in our evolutionary sequences. We begin the evolution with the outermost shells at a radius extending to $10^{10} \mathrm{~cm}$ (Figure

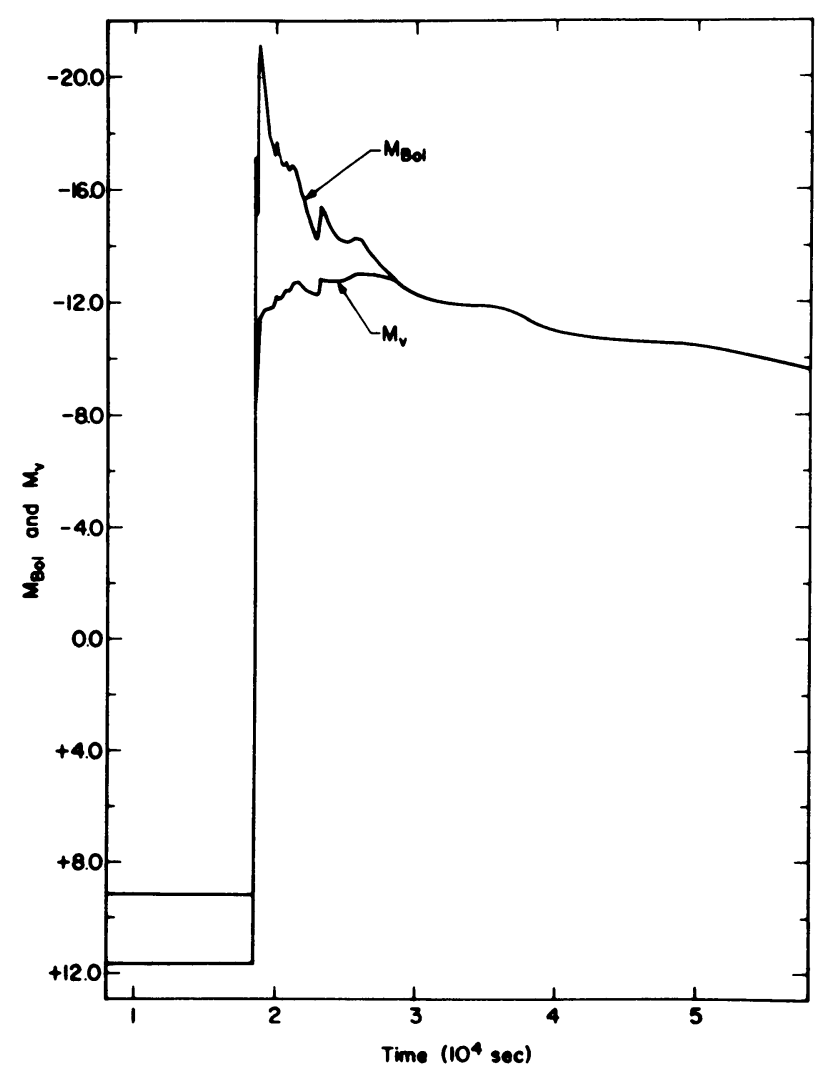

Fig. 6. The light curve as a function of time for a model with extremely enhanced carbon in the envelope. 
7) and then allow these shells to fall back onto the white dwarf at various rates. We have studied only two models so far. In one, we let the material fall back onto the white dwarf at the beginning of the evolution; in the other, we waited until the shell source temperature had reached 129 million deg before turning on the infall. We find that these two models, the first emphasizing the effects of the recently accreted material and the second the effects of the mass inflow on the evolution, also reproduce the gross features of the nova outburst. However, while the light curves are in better agreement with the observations (Figure 8, Figure 9) the amount of material ejected is smaller than in the models without infall. Therefore, to eject the same amount of mass as in the earlier studies, we require more CNO nuclei in the envelope. This is because the energy produced in the runaway must not only go to ejecting the envelope, but also must halt the currently infalling material and turn it around. In addition, the $\beta^{+}$-unstable nuclei produced in the interior do not penetrate into the infalling material, so that all of the heating (which causes the ejection) occurs in the deeper layers. Therefore, the outermost material in the expanding shell will not show evidence of enhanced CNO nuclei.

\subsection{SUMMARY}

It seems clear that our published work has demonstrated that a thermonuclear runaway in the hydrogen envelope of a carbon white dwarf is currently the most viable mechanism for the classical nova outburst. Our studies show that such an object can eject $10^{-4} M_{\odot}$ into the interstellar medium moving with speeds up to $100000 \mathrm{~km} \mathrm{~s}^{-1}$. Our light curves resemble those observed during the outburst of a fast nova although there are still some

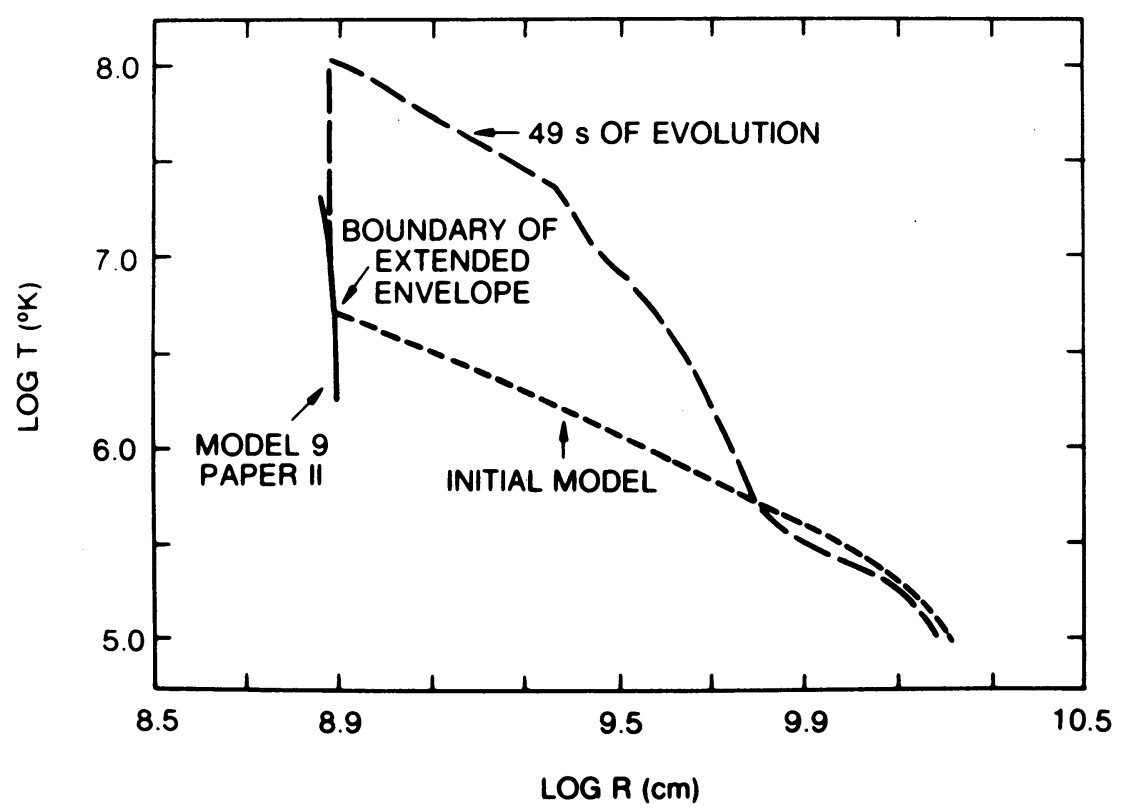

Fig. 7. The temperature in the envelope as a function of radius. The curve labeled 'Model 9' refers to a model from Paper II. The curve labeled 'initial model' is the equilibrium model for the accretion studies with the gravity reduced artificially for the outermost zones. The upper dashed curve shows the accretion model after $49 \mathrm{~s}$ of evolution. 


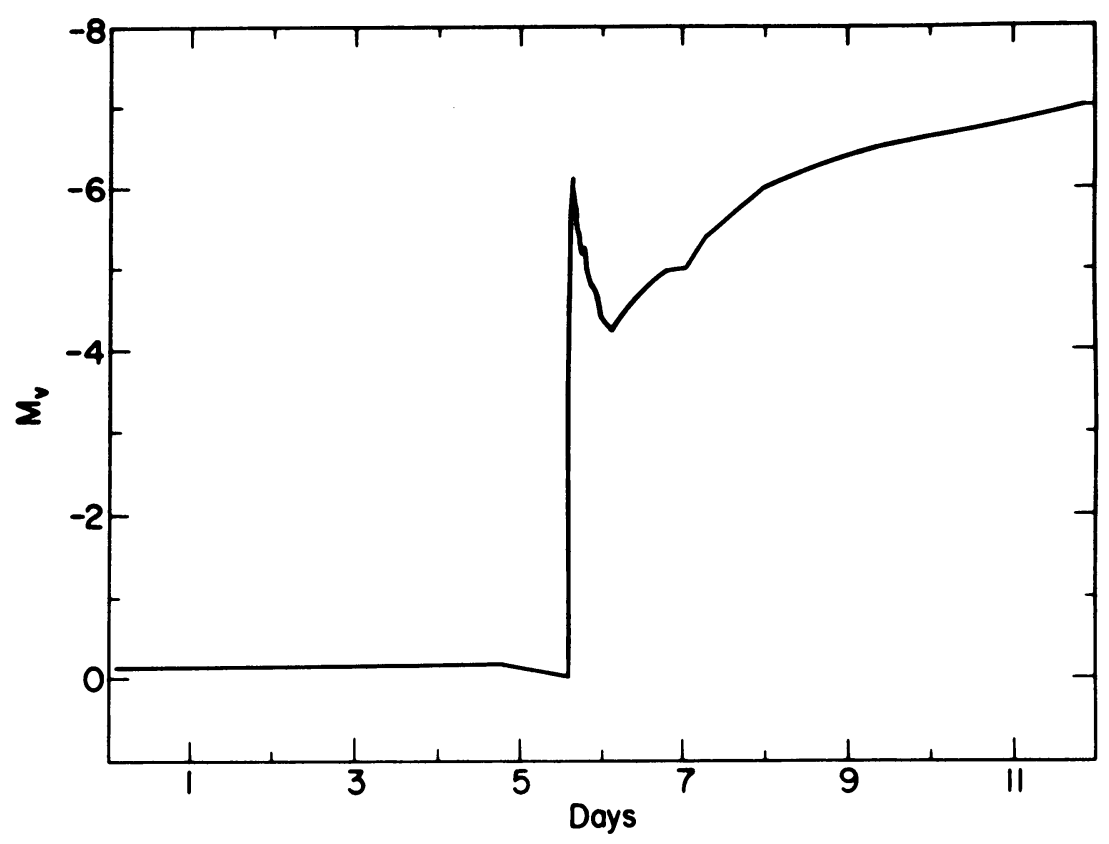

Fig. 8. The light curve for an accretion model where accretion begins at time zero of the evolution.

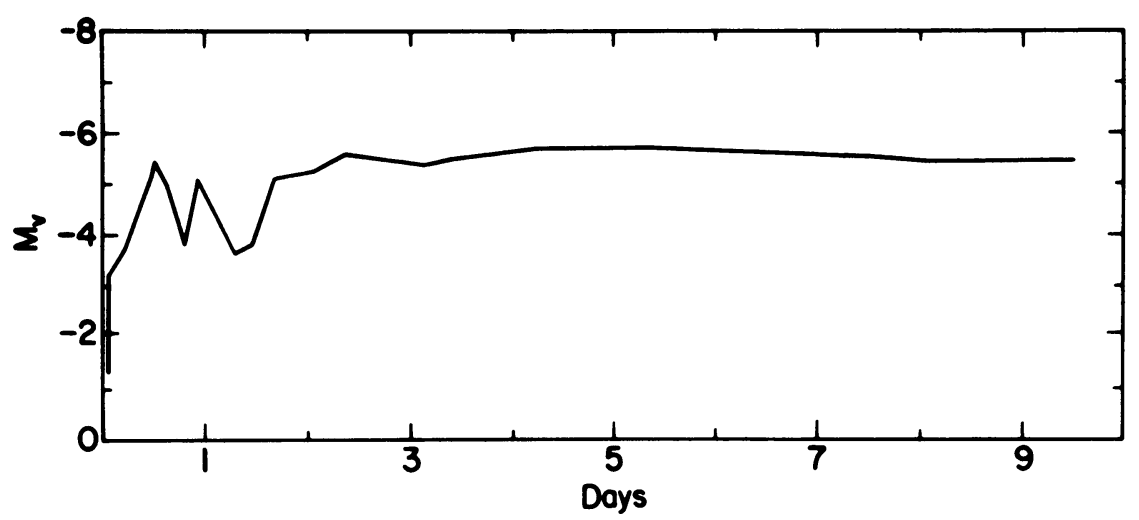

Fig. 9. The light curve for a model where accretion doesn't begin until nearly peak temperature in the shell source.

discrepent features which are probably caused by our neglect of the accretion disc and circumstellar material surrounding the binary.

A very important result has been our prediction that the outburst is dependent upon the envelope having enhanced CNO nuclei in the shell. The reason for this is based upon the behavior of the $\beta^{+}$-unstable nuclei since they become the most abundant of the CNO nuclei in the envelope during the major stages of the outburst. The most interesting implication of the enhanced abundances is our prediction that the nuclei will be enhanced 
in the ejected nebulae and recent observations are now providing verification of this prediction.

It is also encouraging that our models provide us with a possible explanation for the source of the material that is ejected for long periods after the initial outburst. Up to now it has not been clear how a purely explosive event could explain this process. In fact, it turns out there are two phases to a classical nova outburst - the initial hydrodynamic ejection phase and the later hydrostatic equilibrium phase. In the fast nova the initial phase is more important while the reverse is true for the slow novae. If our hypothesis that the diffuse enhanced and orion spectra are produced in the hydrostatic remnant proves to be correct, then further studies of these absorption systems will be very useful in determining the characteristics and evolution of the hydrostatic remnant.

Although we now understand the basic mechanism of the nova outburst, there are a number of very important studies that should be performed on our basic models. A much more elaborate parameter study of the initial chemical abundances is needed since we have only studied models resulting from the initial outburst on a white dwarf. In succeeding outbursts, ${ }^{14} \mathrm{~N}$ and ${ }^{15} \mathrm{~N}$ will be enhanced as well which will change some of the features of the outburst. For example, some of these models should produce shock ejected material, a rather common feature in the outbursts of the classical nova (Sparks, 1969).

We shall, of course, continue our accretion studies and our studies with smaller envelope masses. In both cases we are going in the direction of including the most recent ideas concerning the structure of the pre-outburst object. We have already proposed that the accretion disc is responsible for the oval shape of the ejected material (Sparks and Starrfield, 1973) and it will be very interesting to include its effects in the hydrodynamic evolution. It will also be interesting, in connection with the studies of chemical evolution in the galaxy, to determine in some detail what nuclei are produced in the nova outburst and ejected into the interstellar medium.

Finally, it will be useful to continue our studies of accretion onto massive white dwarfs $\left(M>1.3 M_{\odot}\right)$ as it is these objects that may be related to the formation of neutron stars in close binary systems.

\section{Acknowledgements}

The authors are grateful to the many people who have contributed to the progress of this work by their comments, criticism, and suggestions. We thank Jeff Colvin, Jay Gallagher, Don Lamb, and Hugh Van Horn for informing us of their results prior to publication. J. W. Truran and S. Starrfield thank the National Science Foundation for support under their respective grants MPS73-05117 and MPS73-05271. In addition S. Starrfield is pleased to thank Dr Peter Carruthers for the hospitality of the Los Alamos Scientific Laboratory and a generous allotment of computer time. He also thanks Arizona State University for a Faculty Grant-in-aid and a generous allotment of computer time.

\section{Bibliography}

Allen, C. W.: 1963, Astrophysical Quantities, Athlone Press, London.

Antipova, L. I.: 1974, in G. Contoupolous (ed.), IAU Highlights of Astronomy, D. Reidel, Dordrecht, Holland, p. 501.

Bath, G. T.: 1974, Monthly Notices Roy. Astron. Soc. 171, 311.

Clayton, D. D.: 1968, Principles of Stellar Evolution and Nucleosynthesis, McGraw-Hill, New York. 
Cloutman, L. D. and Starrfield, S.: 1975 (in preparation).

Cox, A. N. and Stewart, J. N.: 1965, Astrophys. J. Suppl. 11, 22.

Gallagher, J. S. and Code, A. D.: 1974, Astrophys. J. 189, 303.

Gallagher, J. S. and Starrfield, S.: 1976, Monthly Notices Roy. Astron. Soc., in press.

Geisel, S. L., Kleinmann, D. E. and Low, F. J.: 1970, Astrophys. J. Lett. 161, L101.

Giannone, P. and Weigert, A.: 1967, Z. Astrophys. 67, 41.

Hazelhurst, J.: 1962, Adv. Astron. Astrophys. $1,1$.

Herbst, W., Hesser, J. E., and Ostriker, J. P.: 1974, Astrophys. J. 193, 679.

Hoyle, F. and Clayton, D. D.: 1974, Astrophys. J. 191, 705.

Kraft, R. P.: 1964, Astrophys. J. 139, 457.

Kutter, G. S. and Sparks, W. M.: 1972, Astrophys. J. 175, 407.

Lamb, D. W. and Van Horn, H. M.: 1975, Astrophys. J. (in press).

Lucy, L. B. and Solomon, P.: 1970, Astrophys. J. 159, 879.

Paczynski, B.: 1971, Ann. Rev. Astron. Astrophys. 9, 183.

Paczynski, B.: 1976, these proceedings, p. 75.

Payne-Gaposchkin, C.: 1957, The Galactic Novae, Dover Press, New York.

Pottasch, S.: 1958, Ann. Astrophys. 22, 412.

Redkoborodyi, Y. N.: 1972, Astrofizika 8, 261.

Rose, W. K.: 1968, Astrophys. J. 152, 245.

Rose, W. K. and Smith, R.: 1972, Astrophys. J. 172, 699.

Ruusalepp, M. and Luud, L.: 1970, Tartu Obs. Publ. 39, 89.

Smak, J.: 1971 in IAU Coll. 15, Bamberg, p. 248.

Sneden, C. and Lambert, D. L.: 1975, Monthly Notices Roy. Astron. Soc. 170, 533.

Sparks, W. M.: 1969, Astrophys. J. 158, 569.

Sparks, W. M. and Starrfield, S.: 1973, Monthly Notices Roy. Astron. Soc. 164, 1P.

Sparks, W. M., Starrfield, S., and Truran, J. W.: 1976, Astrophys. J., in press.

Starrfield, S.: 1970, Astrophys. J. 161, 361.

Starrfield, S., Sparks, W. M., and Truran, J. W.: 1974a, Astrophys. J. Suppl. 261, 28, 247 (Paper II).

Starrfield, S., Sparks, W. M., and Truran, J. W.: 1974b, Astrophys. J. 192, 647 (Paper III).

Starrfield, S., Sparks, W. M., and Truran, J. W.: 1974c, 19 th Liege Coll., in press (Paper IV).

Starrfield, S., Sparks, W. M., and Truran, J. W.: 1974d, 19th Liege Coll., in press (Paper VI).

Starrfield, S., Truran, J. W., and Sparks, W. M.: 1975, Astrophys. J. Lett. 198, L1 13 (Paper V).

Starrfield, S., Trurah, J. W., Sparks, W. M., and Kutter, G. S.: 1972, Astrophys. J. 176, 169 (Paper I).

Swedlund, J. B., Kemp, J. C. and Wolstencroft, R. D.: 1974, Astrophys. J. Lett. 193, L11.

Taam, R. and Faulkner, J.: 1975, Astrophys. J. (in press).

Van Horn, H. M. and Colvin, J.: 1975 (in preparation).

Walker, M. F. and Chincarini, G.: 1968, Astrophys. J. 154, 157.

\section{DISCUSSION}

Shaviv: If the hydrogen convective zone penetrates into the core, you cannot explain the existance of DA's. Concerning the outer boundary condition, we know that (a) the accreting matter establishes a shock around the white dwarf and (b) the results are sensitive to the boundary conditions. Consequently it is difficult to know how closely your very simplified condition simulates reality.

Starrfield: The convective zone is for a pure carbon white dwarf. The addition of hydrogen to the surface will probably shut off convection after a certain amount of hydrogen has been transferred. On your second comment: as I said in my talk the hydrostatic accretion studies will only change the time scale and should not effect the basic characteristic of the Nova. I agree that the results are sensitive to the boundary conditions in our accretion studies. Unfortunately, our code limits us to methods in which we do not rezone the outer envelope. We hope to improve this in the future.

Faulkner: I would like to make two points about some work which you referred to by Taam and myself. These points arise out of the different methods we employed. I think they are important, but perhaps more so for the lower rates of accretion which interested us.

(1) Since we construct complete stellar models, our 'lower boundary conditions' are $R, M=0$. In your work, as I understand it, no heat leakage occurs below the thin outer regions you study. As a result, energy is bottled up and timescales are considerably shortened. In contrast, we find that significant heat transfer occurs down to at least $M_{r} \sim 0.9 M_{\odot}$ in our $1 M_{\odot}$ model.

(2) Results from perturbing a purely static initial model may be misleading. We find burning starts in very different regions from those in which it would occur in a corresponding static model, as the long accretion times result in PdV work which is retained while some flows inwards and outwards. Taam and I accreted material directly, as is very convenient using Eggleton's code. 